\title{
Enhanced oral bioavailability of vancomycin in rats treated with long-term parenteral nutrition
}

\author{
Keizo Fukushima', Akira Okada', Yoriko Hayashi' ${ }^{1}$, Hideki Ichikawa ${ }^{2}$, Asako Nishimura ${ }^{3}$, Nobuhito Shibata ${ }^{3}$ \\ and Nobuyuki Sugioka ${ }^{1 *}$
}

*Correspondence: nsugioka@pharm. kobegakuin.ac.jp

${ }^{1}$ Department of Clinical Pharmacokinetics, Faculty of Pharmaceutical Sciences, Kobe Gakuin University, Chuo-ku, Kobe 650-8586, Japan

Full list of author information is available at the end of the article

\begin{abstract}
Long-term parenteral nutrition (PN) can induce intestinal atrophy, leading to a loss of epithelial integrity in the small intestines. This change may alter the intestinal permeability of vancomycin (VCM), a non-absorbable antibiotic. The aim of the present study was to investigate the effect of PN on the pharmacokinetics of VCM in rats. VCM was intravenously $(5 \mathrm{mg} / \mathrm{kg}$ ) or intraduodenally $(20 \mathrm{mg} / \mathrm{kg}$ ) administered to control and PN rats, which were prepared by administration of PN for 9 days. After intravenous administration, there were no significant differences in any of the VCM pharmacokinetic parameters between the control and PN rats. However, after intraduodenal administration, the maximum concentration and area under the plasma concentration-time curve of VCM in PN rats was approximately 2.4- and 2.6-fold higher, respectively, than in the control rats; the calculated bioavailability was approximately 0.5 and $1.3 \%$ in control and PN rats, respectively. These results indicated that PN administration did not affect VCM disposition, but enhanced VCM absorption; however, the enhanced oral VCM bioavailability was statistically, not clinically, significant. Therefore, while long-term PN administration may play a role in the enhancement of VCM bioavailability, this effect may be negligible without any complications.
\end{abstract}

Keywords: Vancomycin, Absorption, Bioavailability, Pharmacokinetics, Parenteral nutrition, Intestinal atrophy

\section{Background}

Parenteral nutrition (PN) serves as a critical therapy for patients in conditions where oral ingestion is not adequate. In spite of its usefulness, there are also many possible complications, such as catheter-related bloodstream infection (Hvas et al. 2014), impaired glucose tolerance (Beltrand et al. 2007), and parenteral nutrition-associated liver disease (Nandivada et al. 2013). In addition, long-term PN administration can induce intestinal atrophy; the reduction of the mucosal barrier may mediate bacterial translocation (BT), in which the intestinal bacteria and/or their toxic products invade the bloodstream and induce production of inflammatory cytokines, subsequently leading to sepsis and multiple organ failure (Hatakeyama and Matsuda 2014; Li et al. 1999; Sun et al. 2006).

\section{Springer}

(C) 2015 Fukushima et al. This article is distributed under the terms of the Creative Commons Attribution 4.0 International License (http://creativecommons.org/licenses/by/4.0/), which permits unrestricted use, distribution, and reproduction in any medium, provided you give appropriate credit to the original author(s) and the source, provide a link to the Creative Commons license, and indicate if changes were made. 
Selective decontamination of the digestive tract (SDD) prophylaxis of BT, which prevents the overgrowth of pathogens by enteral co-administration of non-absorbable antibiotics, such as colistin, tobramycin, amphotericin B, and vancomycin (VCM) (Benus et al. 2010; Cerdá et al. 2007; Roos et al. 2011) has been previously reported. It has been shown that any absorption of the non-absorbable antibiotic in the SDD regimen can be considered negligible under "normal conditions"; however, significant absorption of tobramycin was reported in critically ill patients (Oudemans-van Straaten et al. 2011), as was VCM in patients with chemotherapy-associated and/or Clostridium difficile colitis (Aradhyula et al. 2006; Bergeron and Boucher 1994). In addition, we previously reported the enhanced intestinal permeability of a hydrophilic dye, phenolsulfonphthalein, in PNinduced intestinal atrophy (Fukushima et al. 2015). On the basis of these findings, there is potential to enhance the absorption of typically non-absorbable antibiotics in patients with PN-induced intestinal atrophy. It is well known that trough concentrations of VCM are associated with its nephrotoxicity (Elyasi et al. 2012) and that the rapid infusion of VCM induces red man syndrome (Healy et al. 1990). Generally, the absorption via passive diffusion of a hydrophilic drug is rapid. Therefore, there are concerns about side effects caused by the systemic exposure after enteral administration of VCM.

The aim of the present study was to investigate the impact of PN-induced intestinal atrophy on the absorption of VCM and to assess its clinical significance. Based on the usage of VCM in SDD regimen, VCM was administered intraduodenally to rats administered PN, and the absorption and disposition of $\mathrm{VCM}$ were assessed with intravenous administration.

\section{Methods}

Materials and animals

An injectable formulation of vancomycin (VCM) hydrochloride was purchased from Shionogi Co., Ltd., (Osaka, Japan). PNTWIN ${ }^{\circledR}$ No.3 and VITAJECT ${ }^{\circledR}$ were purchased from Ajinomoto Pharmaceuticals Co., Ltd. (Tokyo, Japan) and Terumo Co., Ltd. (Tokyo, Japan), respectively. Ampicillin and bupivacaine were obtained from Meiji Seika Pharma Co., Ltd. (Tokyo, Japan) and AstraZeneca PLC (London, UK), respectively. All other reagents were of analytical grade and were used without further purification. Male Wistar rat (weighing $250 \pm 10$ g, 10-weeks old) were purchased from Nippon SLC Co., Ltd. (Hamamatsu, Japan). All animal experiments in the present study were approved by the Animal Experimentation Committee of Kobe Gakuin University (approval number: A14-31). Rats had free access to food and water and were acclimated in a temperaturecontrolled facility with a $12 \mathrm{~h}$ light/dark cycle for at least 3 days before use.

\section{Preparation of PN-administered rats and laboratory tests}

Parenteral nutrition-administered rats ( $\mathrm{PN}$ rats) were prepared by the same method in our previous report (Fukushima et al. 2015) with only a change in the duration of PN administration; the preparation scheme of the PN rats are shown in Scheme 1. Briefly, 3 days before the start of PN administration, cardiac catheterization was performed via the right jugular vein with a polyurethane catheter (0.6-mm ID, 0.9-mm OD, Primetech Co., Tokyo, Japan) in rats anesthetized with sodium pentobarbital, and given ampicillin for prevention of infection and local bupivacaine for pain relief. The cannulated rats 


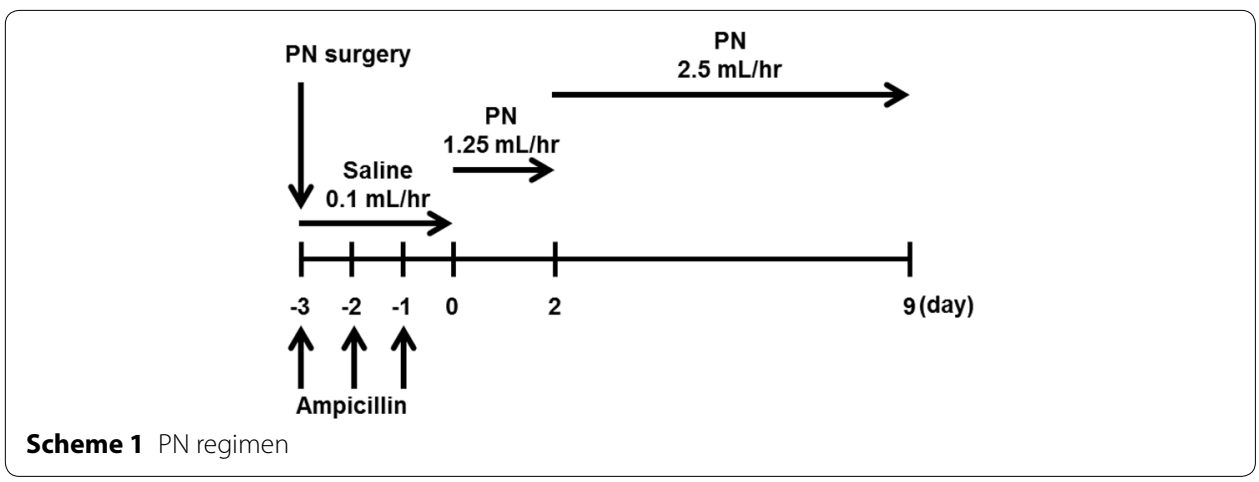

were housed individually in cages with free access to food and water, and received saline at a rate of $0.1 \mathrm{~mL} / \mathrm{h}$ for 3 days via the catheter with a syringe pump (ISIS Co., Ltd., Osaka, Japan), and daily ampicillin ( $2 \mathrm{mg} / \mathrm{kg}, 2$-min infusion) for recovery from surgery. For PN administration, infusion of PN solution was started at a rate of $1.25 \mathrm{~mL} / \mathrm{h}$ for 2 days under fasting and water-deprived conditions; PN solution consisting of glucose, amino acids, electrolytes, and vitamins (approximately $0.97 \mathrm{kcal} / \mathrm{mL}$ ) was prepared by mixing PNTWIN No. 3 with VITAJECT (Fukushima et al. 2015). Subsequently, PN administration was performed at a rate of $2.5 \mathrm{~mL} / \mathrm{h}$ (viz., $60 \mathrm{~mL} /$ day, $58 \mathrm{kcal} /$ day) for 7 days; likewise, the sham operated rats (control rats) underwent the same regimen with saline and allowed free access to food throughout the treatment. All rats were fasted overnight before the VCM pharmacokinetic study, and blood samples for laboratory testing were taken just before VCM administration; total protein (TP), serum albumin level (Alb), total cholesterol (T-Cho), triglyceride (TG), aspartate transaminase (AST), alanine transaminase (ALT), blood urea nitrogen (BUN), and serum creatinine (CRE) were measured by a commercial laboratory, Oriental Yeast Co., Ltd. (Tokyo, Japan).

\section{Intravenous and intraduodenal administration of VCM}

The fasted control and PN rats were allocated to two groups ( $\mathrm{n}=4$ /group) based on the administration route: intravenous and intraduodenal VCM administration. In the intravenous administration study, rats were anesthetized with sodium pentobarbital (50 $\mathrm{mg} / \mathrm{kg}$ ), and the VCM saline solution $(2.5 \mathrm{mg} / \mathrm{mL})$ was bolus injected into the left femoral vein $(5 \mathrm{mg} / \mathrm{kg})$. Blood samples were taken $5,15,30,45,60,90,120,180,240$, and $360 \mathrm{~min}$ after administration, and were centrifuged at 12,000 rpm for $15 \mathrm{~min}$ to collect the plasma fraction. In the intraduodenal administration study, the abdominal cavity was opened in anesthetized rats and the stomach was exposed. A small incision was made on the lesser curvature of the stomach, and the VCM saline solution $(10 \mathrm{mg} / \mathrm{mL})$ was administered through the incision and the pylorus into the duodenum $(20 \mathrm{mg} / \mathrm{kg})$ using a sterilized oral feeding needle. After administration, the incision was closed with a tissue adhesive and the pylorus was ligated. Blood samples were taken 5, 15, 30, 60, 90, 120, $180,240,300$, and $360 \mathrm{~min}$ after administration, and plasma samples were collected by centrifugation. The collected plasma samples in both studies were immediately frozen at $-80^{\circ} \mathrm{C}$ until analysis. 


\section{VCM assay}

The VCM assay was performed by a previously reported liquid chromatography/tandem mass spectrometry (LC/MS/MS) method (Shibata et al. 2003) with some modifications; briefly, a $100 \mu \mathrm{L}$ plasma sample was added to $60 \mu \mathrm{L}$ of $30 \%$ trifluoroacetic acid to precipitate protein. After vortexing and centrifugation at 12,000 rpm for $15 \mathrm{~min}$, the supernatant was diluted in $340 \mu \mathrm{L}$ of distilled water and was passed through a filter, and $10 \mu \mathrm{L}$ of the filtrate was injected into a Quattro Ultima LC/MS/MS system with a 2690 Separation Module (Waters Co, MA, UK). VCM separation was performed with a QUICKSORB ODS column (i.d. $2.1 \mathrm{~mm} \times 100 \mathrm{~mm}, 3 \mu \mathrm{m}$, Chemco Scientific Co., Ltd., Osaka, Japan), and the elution was carried out isocratically at a flow rate of $0.2 \mathrm{~mL} / \mathrm{min}$ with the degassed mobile phase, acetonitrile: $0.1 \%$ acetic acetate (2:8). Mass spectrometry was conducted with electrospray ionization in positive mode (ESI+) under the following conditions: source temperature, $130{ }^{\circ} \mathrm{C}$; cone voltage, $35 \mathrm{~V}$; capillary voltage, $4.0 \mathrm{kV}$. VCM intensity was monitored by multiple reaction monitoring (MRM) with $18 \mathrm{eV}$ of collision energy for the VCM transition $(725-144 \mathrm{~m} / z)$. VCM concentration was quantified by calculating peak area against calibrated samples. The lower limit of quantitation for $\mathrm{VCM}$ was $<0.005 \mu \mathrm{g} / \mathrm{mL}$.

\section{Pharmacokinetic analysis}

A one- or two-compartment model with first-order absorption process was applied to the plasma concentration profiles of VCM; on the basis of the Akaike's Information Criteria (AIC), the latter model was selected in the present study. All pharmacokinetic analyses were performed using WinNonlin ${ }^{\circledR}$ Version 6.3 (Pharsight, Mountain View, $\mathrm{CA})$. The structural parameters, including the absorption rate constant $(k a)$, distribution volume of the central compartment $\left(V_{c}\right)$, distribution volume of the peripheral compartment $\left(V_{p}\right)$, total body clearance $\left(C L_{t o t}\right)$, and distribution clearance (CLD2), and the secondary parameters including the maximum concentration $\left(C_{\text {max }}\right)$, time to maximum concentration $\left(T_{\max }\right)$, distribution half-life $\left(t_{1 / 2 \alpha}\right)$, elimination half-life $\left(t_{1 / 2 \beta}\right)$, and the area under the plasma concentration-time curve $(A U C)$ were calculated. The bioavailability $(F)$ was calculated using the following equation:

$$
F=\left(A U C_{\text {intraduodenal }} / \text { Dose }_{\text {intraduodenal }}\right) /\left(A U C_{\text {intravenous }} / \text { Dose }_{\text {intravenous }}\right),
$$

where Dose represents the administered dosage.

\section{Statistical analyses}

All values are represented by the mean \pm standard error (SE). Statistical differences of the means were assumed to be significant when $p<0.05$ by the Mann-Whitney $\mathrm{U}$ test.

\section{Results}

Body weight and laboratory test results of the control and PN rats are shown in Additional file 1: Table S1. Body weight, TP, and Alb were slightly, but significantly, decreased in PN rats (approximately 88, 82 and 86 \% of control, respectively), and TG levels in PN rats were markedly decreased, approximately $28 \%$ of control. The plasma concentration profiles of VCM after intravenous and intraduodenal administration are shown in Figs. 1 and 2, respectively. After intravenous administration, the plasma concentration profiles 


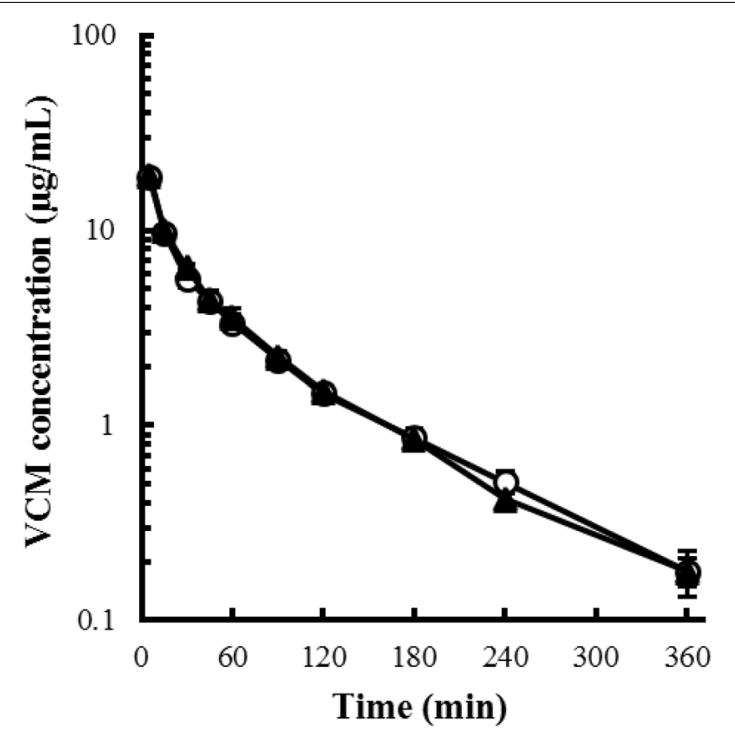

Fig. 1 Plasma concentration profiles of VCM after intravenous administration ( $5 \mathrm{mg} / \mathrm{kg}$ ) to (open circles) control and (filled triangles) PN rats. Each symbol with a bar represents the mean $\pm \mathrm{SE}$ of 4 rats

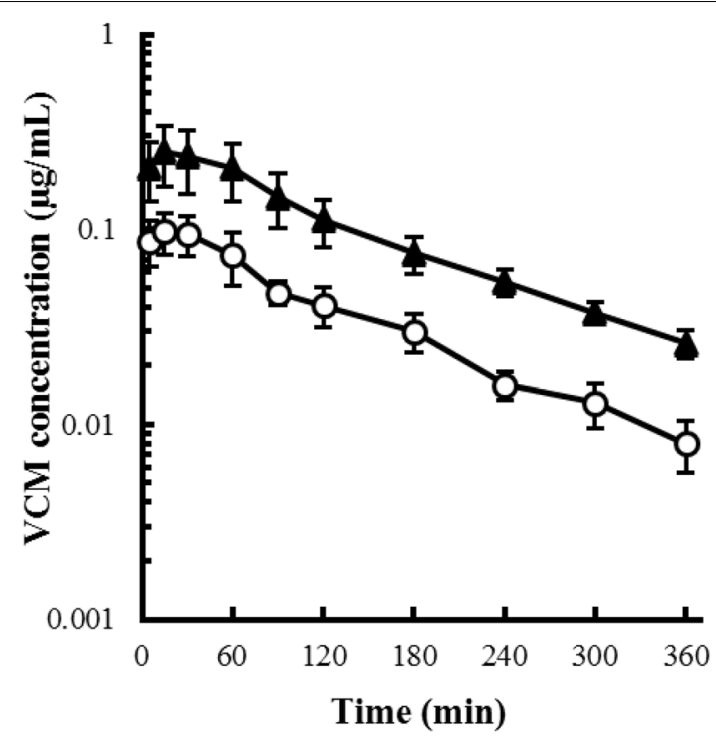

Fig. 2 Plasma concentration profiles of VCM after intraduodenal administration ( $20 \mathrm{mg} / \mathrm{kg}$ ) to (open circles) control and (filled triangles) PN rats. Each symbol with a bar represents the mean \pm SE of 4 rats

of both control and PN rats were similar, and declined in a biphasic manner; after intraduodenal administration, the concentration of VCM in control and PN rats reached a peak within $30 \mathrm{~min}$ and then gradually decreased. The mean VCM concentration in PN rats was consistently higher than that in control rats after intraduodenal administration. The pharmacokinetic parameters of VCM are shown in Additional file 1: Table S2. After intravenous administration, there were no significant differences in any of the pharmacokinetic parameters between the control and PN rats; however, after intraduodenal administration, the $A U C$ in $\mathrm{PN}$ rats was approximately 2.6-fold higher than that in 
control rats, with no other significant differences in the other pharmacokinetic parameters except for $C L_{t o t} / F$. The calculated bioavailability $(F)$ of VCM in the control and PN rats was approximately 0.5 and $1.3 \%$, respectively; the $F$ of VCN in PN rats was approximately 2.6 -fold higher than in control rats.

\section{Discussion and conclusions}

The present study investigated the effect of long-term PN on VCM absorption. In order to more adequately induce intestinal atrophy, the period of PN administration was prolonged by 2 days compared with our previously reported method (Fukushima et al. 2015). The changes in body weight and laboratory test results in the present PN rats were similar to those previously reported, except for no obvious hepatic and renal impairment (Additional file 1: Table S1). Therefore, although no visual inspection of the intestines was performed in the present study, intestinal atrophy was very likely induced in the PN rats of the present study.

After intravenous administration, there were no significant differences in VCM pharmacokinetic parameters, including $A U C$, between the control and PN rats (Fig. 1; Additional file 1: Table S2). Because most of the VCM after intravenous administration is eliminated from the kidneys non-metabolized, not only kidney function but also the binding rate of VCM to plasma proteins may have significant effects on VCM distribution. However, the present results indicated that the decrease in plasma protein observed in PN rats did not significantly affect the distribution and elimination of VCM, possibly due to the relatively low protein binding rate of VCM, which was reported to be approximately 47 \% (Kusama et al. 1998). Additionally, there was no obvious renal impairment observed in PN rats (Additional file 1: Table S1). Therefore, PN administration in the present study did not alter the distribution and elimination of VCM.

After intraduodenal administration, the $C_{\text {max }}$ and $A U C$ of VCM were increased in PN rats, and the bioavailability $(F)$ of VCM showed a 2.6-fold increase in PN rats (Additional file 1: Table S2). Generally, a hydrophilic drug such as VCM is absorbed from the intestines via a paracellular pathway, which is modulated by tight junctions (Del Vecchio et al. 2012; Prasad et al. 2003). We previously reported the loss of intestinal epithelial integrity with decreases in total wet weight, mucosal protein content, and villous height of the intestines in rats administered PN for 7 days (Fukushima et al. 2015), and Sun et al. (2008) also reported a decrease in expression of tight junction proteins, such as occluding, zonula occludens and claudin, in PN-administered mice. Although the details of the mechanism underlying these observations still remain unclear, growing evidence indicates that long-term PN administration induces the disintegration of tight junctions along with intestinal atrophy (Feng et al. 2012; Yang et al. 2009). In addition, the slight hypoproteinemia and marked hypotriglyceridemia were induced by the present PN regimen (Additional file 1: Table S2), possibly due to the long-term treatment without lipid emulsion. Combined with the results of the present study, the enhanced oral bioavailability of VCM may have been due to the disruption of tight junctions caused by malnutrition.

The enhanced oral bioavailability of VCM by long-term PN administration was statistically, but not clinically, significant; the VCM bioavailability of $1.3 \%$ (Additional file 1: Table S2) in the present PN rats would be negligible in clinical settings. However, 
it should be noted here that the PN rats in this study were relatively "healthy" and free from any complications such as renal impairment, colitis, and bacterial translocation (BT). Systemic exposure of VCM may be further enhanced with these complications, particularly in BT, in which bacteria and/or its toxins, far larger than VCM, permeate the intestinal mucosa. Nonetheless, the absorption of VCM itself is not necessarily an unfavorable feature in BT, and may possibly be utilized as a tracer for invaded bacteria. Therefore, the present results demonstrated that long-term PN enhances the oral bioavailability of VCM; however, its clinical significance requires further evaluation.

In conclusion, long-term PN administration did not significantly affect the disposition of VCM, but increased VCM oral bioavailability, possibly due to a loss of tight junction integrity along with intestinal atrophy. The enhanced VCM oral bioavailability was statistically, but not clinically, significant. Therefore, while long-term PN administration plays a role in the enhancement of VCM bioavailability, this effect may be negligible without any complications.

\section{Additional file}

Additional file 1: Table S1. Body weight and laboratory test results of control and PN rats. Table S2. Pharmacokinetic parameters of VCM after intravenous $(5 \mathrm{mg} / \mathrm{kg})$ and intraduodenal (20 mg/kg) administration to control and PN rats

Authors' contributions

$\mathrm{HI}, \mathrm{AN}$, Nobuhito S and Nobuyuki S made substantial contributions to conception and design of the present study. KF, $\mathrm{AO}$ and $\mathrm{YH}$ made substantial contributions to acquisition and interpretation of data. All authors reviewed and approved the final manuscript.

\section{Author details}

${ }^{1}$ Department of Clinical Pharmacokinetics, Faculty of Pharmaceutical Sciences, Kobe Gakuin University, Chuo-ku, Kobe 650-8586, Japan. ${ }^{2}$ Department of Physical Pharmacy, Faculty of Pharmaceutical Sciences, Kobe Gakuin University, Chuo-ku, Kobe 650-8586, Japan. ${ }^{3}$ Department of Biopharmaceutics, Faculty of Pharmaceutical Science, Doshisha Women's College of Liberal Arts, Kyotanabe, Kyoto 610-0395, Japan.

\section{Acknowledgements}

The present study was supported by a Japan Society for the Promotion of Science (JSPS) Grant-in-Aid for Young Scientists (B), Grant Number 24790178.

\section{Compliance with ethical guidelines}

\section{Competing interests}

The authors declare that they have no competing interests.

Received: 3 April 2015 Accepted: 10 August 2015

Published online: 22 August 2015

\section{References}

Aradhyula S, Manian FA, Hafidh SA, Bhutto SS, Alpert MA (2006) Significant absorption of oral vancomycin in a patient with clostridium difficile colitis and normal renal function. South Med J 99(5):518-520

Beltrand J, Colomb V, Marinier E, Daubrosse C, Alison M, Burcelin R, Cani PD, Chevenne D, Marchal CL (2007) Lower insulin secretory response to glucose induced by artificial nutrition in children: prolonged and total parenteral nutrition. Pediatr Res 62(5):624-629

Benus RF, Harmsen HJ, Welling GW, Spanjersberg R, Zijlstra JG, Degener JE, van der Werf TS (2010) Impact of digestive and oropharyngeal decontamination on the intestinal microbiota in ICU patients. Intensive Care Med 36(8):1394-1402

Bergeron L, Boucher FD (1994) Possible red-man syndrome associated with systemic absorption of oral vancomycin in a child with normal renal function. Ann Pharmacother 28(5):581-584

Cerdá E, Abella A, de la Cal MA, Lorente JA, García-Hierro P, van Saene HK, Alía I, Aranguren A (2007) Enteral vancomycin controls methicillin-resistant Staphylococcus aureus endemicity in an intensive care burn unit: a 9-year prospective study. Ann Surg 245(3):397-407 
Del Vecchio G, Tscheik C, Tenz K, Helms HC, Winkler L, Blasig R, Blasig IE (2012) Sodium caprate transiently opens claudin5-containing barriers at tight junctions of epithelial and endothelial cells. Mol Pharm 9(9):2523-2533

Elyasi S, Khalili H, Dashti-Khavidaki S, Mohammadpour A (2012) Vancomycin-induced nephrotoxicity: mechanism, incidence, risk factors and special populations. A literature review. Eur J Clin Pharmacol 68:1243-1255

Feng Y, Ralls MW, Xiao W, Miyasaka E, Herman RS, Teitelbaum DH (2012) Loss of enteral nutrition in a mouse model results in intestinal epithelial barrier dysfunction. Ann NY Acad Sci 1258:71-77

Fukushima K, Miki T, Nakamoto K, Nishimura A, Koyama H, Ichikawa H, Shibata N, Tokuyama S, Sugioka N (2015) Effect of intestinal atrophy and hepatic impairment induced by parenteral nutrition on drug absorption and disposition in rats. JPEN 39(2):218-227

Hatakeyama N, Matsuda N (2014) Mechanisms of inflammatory response and organ dysfunction: organ-protective strategy by anesthetics. Curr Pharm Des. (Epub ahead of print)

Healy DP, Sahai JV, Fuller SH, Polk RE (1990) Vancomycin-induced histamine release and "red man syndrome": comparison of 1- and 2-hour infusions. Antimicrob Agents Chemother 34:550-554

Hvas CL, Farrer K, Donaldson E, Blackett B, Lloyd H, Forde C, Garside G, Paine P, Lal S (2014) Quality and safety impact on the provision of parenteral nutrition through introduction of a nutrition support team. Eur J Clin Nutr 68(12):1294-1299

Kusama M, Yamamoto K, Yamada H, Kotaki H, Sato H, Iga T (1998) Effect of cilastatin on renal handling of vancomycin in rats. J Pharm Sci 87(9):1173-1176

Li YS, Li JS, Jiang JW, Liu FN, Li N, Qin WS, Zhu H (1999) Glycyl-glutamine-enriched long-term total parenteral nutrition attenuates bacterial translocation following small bowel transplantation in the pig. J Surg Res 82(1):106-111

Nandivada P, Carlson SJ, Chang MI, Cowan E, Gura KM, Puder M (2013) Treatment of parenteral nutrition-associated liver disease: the role of lipid emulsions. Adv Nutr 4(6):711-717

Oudemans-van Straaten HM, Endeman H, Bosman RJ, Attema-de Jonge ME, van Ogtrop ML, Zandstra DF, Franssen EJ (2011) Presence of tobramycin in blood and urine during selective decontamination of the digestive tract in critically ill patients, a prospective cohort study. Crit Care 15(5):R240

Prasad YV, Puthli SP, Eaimtrakarn S, Ishida M, Yoshikawa Y, Shibata N, Takada K (2003) Enhanced intestinal absorption of vancomycin with Labrasol and D-alpha-tocopheryl PEG 1000 succinate in rats. Int J Pharm 250(1):181-190

Roos D, Dijksman LM, Oudemans-van Straaten HM, de Wit LT, Gouma DJ, Gerhards MF (2011) Randomized clinical trial of perioperative selective decontamination of the digestive tract versus placebo in elective gastrointestinal surgery. $\mathrm{Br}$ J Surg 98(10):1365-1372

Shibata N, Ishida M, Prasad YV, Gao W, Yoshikawa Y, Takada K (2003) Highly sensitive quantification of vancomycin in plasma samples using liquid chromatography-tandem mass spectrometry and oral bioavailability in rats. J Chromatogr B Anal Technol Biomed Life Sci 789(2):211-218

Sun X, Spencer AU, Yang H, Haxhija EQ, Teitelbaum DH (2006) Impact of caloric intake on parenteral nutrition-associated intestinal morphology and mucosal barrier function. JPEN 30(6):474-479

Sun X, Yang H, Nose K, Nose S, Haxhija EQ, Koga H, Feng Y, Teitelbaum DH (2008) Decline in intestinal mucosal IL-10 expression and decreased intestinal barrier function in a mouse model of total parenteral nutrition. Am J Physiol Gastrointest Liver Physiol 294(1):G139-G147

Yang H, Feng Y, Sun X, Teitelbaum DH (2009) Enteral versus parenteral nutrition: effect on intestinal barrier function. Ann NY Acad Sci 1165:338-346

\section{Submit your manuscript to a SpringerOpen ${ }^{\circ}$ journal and benefit from:}

- Convenient online submission

- Rigorous peer review

- Immediate publication on acceptance

- Open access: articles freely available online

- High visibility within the field

- Retaining the copyright to your article

Submit your next manuscript at $\boldsymbol{s p r i n g e r o p e n . c o m ~}$ 